\title{
Dominant colour descriptor with spatial information for content-based image retrieval
}

\begin{abstract}
An important problem in colour Content-based Image Retrieval (CBIR) is the lack of an effective way to represent both the colour and spatial information of an image. In order to solve this problem, a new dominant colour descriptor that employs spatial information of image is proposed. A maximum of three dominant colour regions in an image together with their respective coordinates of the Minimum-Bounding Rectangle (MBR) are first extracted using the Colour-based Dominant Region segmentation. The Improved Sub-block technique is then used to determine the location of the dominant colour regions by taking into consideration the total horizontal and vertical distances of a region at each location where it overlaps. A Query-by-Example CBIR system implementing the colour-spatial technique is developed. Experimental studies on an image database consisting of 900 images are conducted. From the experiments, it is evident that retrieval effectiveness has significantly improved by $85.86 \%$.
\end{abstract}

Keyword: Content-based image retrieval (CBIR); Colour; Spatial information; Image; Dominant colour descriptor 